\title{
ANP-FIS Method for Determining the Knowledge Management Strategy
}

\author{
Ahmad Nadali, Hamid Eslami Nosratabadi, and Sanaz Pourdarab
}

\begin{abstract}
The suitable strategy is the critical influential factor on the success of knowledge management (KM) implementation in an organization. The purpose of this research is presenting a hybrid method to determine the knowledge management strategy. Here, a model has been proposed with the use of ANP as one of the MCDM techniques to identify the most suitable knowledge management strategy and a Fuzzy Expert System has been designed to specify the best hybrid dynamic strategy. The presented steps have been run in an Iranian Bank as the empirical study.
\end{abstract}

Index Terms - Knowledge Management Strategy, Dynamic Strategy, Human Oriented Strategy, System Oriented Strategy, Analytic Network Process (ANP), Fuzzy Expert System

\section{INTRODUCTION}

As knowledge is taking on an important strategic role, numerous companies are expecting their knowledge management (KM) to be performed effectively in order to leverage and transform the knowledge into competitive advantages [1]. The effective KM largely begins with a proper KM strategy. Hence, in order to implement the KM successfully, there is a critical issue of how companies can better evaluate and select a favorable KM strategy. Generally, selecting what kinds of KM strategies to use depends on the different purposes, the limited resources, and even the preferences of companies [1]. As to alternatives of KM strategy, [2] notes two types of KM strategies: the codification strategy (seeking to document and store knowledge in databases) and the personalization strategy (seeking to develop networks of people for communicating ideas).Choi and Lee [3] examined 54 Korean companies and categorized their KM strategies into passive, system-oriented, human-oriented, and dynamic which focusing on both knowledge reusability through IT and knowledge sharing through informal discussions among employees - was found to result in higher performance. Schulz and Jobe [4] developed four categories of KM strategies - codification, tacit, focused, and unfocused. Choi and Lee's work, developed on the basis of the knowledge-based view (KBV),

Manuscript received June 1, 2011.

First Author, Ahmad Nadali is with the Department of Information Technology Management, Science and Research Branch, Islamic Azad University, Tehran, Iran (Email:Nadali.ahmad@gmail.com).

Second Author, Hamid Eslami Nosratabadi is with the Department of Information Technology Management, Science and Research Branch, Islamic Azad University, Tehran, Iran (Corresponding author's email is Hamideslami.na@gmail.com).

Third Author, Sanaz Pourdarab is with the Department of Information Technology Management, Science and Research Branch, Islamic Azad University, Tehran, Iran (Email:Pourdarab.sanaz@yahoo.com). which holds that knowledge assets can be a unique resource that may lead to a long-term sustainable competitive advantage [5]. Another research around selecting proper strategy, describes a framework for choosing a knowledge management strategy which is the main output of the CLEVER (cross-sectoral learning in the virtual enterprise) research project in the construction and manufacturing sectors[6].The other one investigates the relationship between knowledge management (KM) strategies and organizational performance and suggest three types of relationship among KM strategies: non-complementarity and non-critical symmetric complementarity, and asymmetric complementarity [7]. Another paper proposes a model to illustrate the link between the strategies and its creating process and the model depicts how companies should align the strategies with four knowledge creation modes such as socialization, externalization, combination, and internalization. It is found that human strategy is effective for socialization while system strategy is effective for combination and suggests that managers should adjust knowledge management strategies in view of the characteristics of their departments[8].Another study develops a forecasting framework based on the fuzzy multi-criteria decision making (FMCDM) approach to help organizations build awareness of the critical influential factors on the success of knowledge management (KM) implementation, measure the success possibility of knowledge management projects, as well as identify the necessary actions prior to embarking on conducting knowledge management[9].The KM strategy selection is a MCDM problem, so it is reasonable to employ MCDM methods to handle it appropriately and ANP is one of the MCDM methods .In a paper,Analytic Network Process (ANP) has been used which is a relatively new MCDM method and can deal with all kinds of interactions systematically and It uses the Decision Making Trial and Evaluation Laboratory (DEMATEL) As the next method which can convert the relations between cause and effect of criteria into a visual structural model and can be used as a way to handle the inner dependences within a set of criteria [10]. In another paper, an effective method based on the ANP has been developed to help companies that need to evaluate and select knowledge management strategies[1]. The ANP has been successfully applied in many fields [11], [12], [13], [14], [15]. No organization can define Its whole strategies as Human-Based or System-Based strategy and run them. The MCDM models cannot always present best results to implement. The most important part for specifying the knowledge management hybrid strategy is identifying the level of each strategy. In our research, this problem has been 
solved via Fuzzy Expert system.

The rest of this paper is structured as follows: In the next section, the overview of the knowledge management strategy, Analytical network process and Fuzzy Expert system are presented. In section III, Research Methodology is described. Section IV will focus on the results and discussion. In the final section, some conclusions are drawn from the study.

\section{LITERATURE REVIEW}

\section{A. Knowledge Management strategy}

Knowledge Management (KM) is often viewed as multidimensional and multidisciplinary which may sometimes lead to a fragmented dialogue on the topic .Also It can be defined as "a process that helps organizations find, select, organize, disseminate and transfer important information and expertise necessary for activities such as problem solving, dynamic learning, strategic planning and decision making". In the other words, it can be considered as dealing with capturing, sharing, applying and creating knowledge in an organization to best leverage this resource internally and externally [16]. The major potential benefits of adopting $\mathrm{KM}$ are well documented in the literature. It represents a potent mechanism to, among others :(i) Enhance decision making through just-in-time intelligence. (ii) Improve work efficiency and productivity. (iii) Increase innovation of products, services and operations. (iv) Improve competency and competitiveness. (v)Enable rapid generation of technical solutions to clients' problems. (vi)Increase responsiveness to customers [16]. KM is the organizational optimization of knowledge to achieve enhanced performance through the use of various methods and techniques. Also, $\mathrm{KM}$ is a systemic way to manage knowledge in the organizationally specified process of acquiring, organizing and communicating knowledge [6]. The purposes of KM vary from organization to organization. For instance, KM is the way to improve an organization's performance, productivity, competitiveness and to promote acquisition, sharing and usage of knowledge. There are many KM purposes such as: to initiate action based on knowledge; to support business strategy implementation; to become an intelligent enterprise; to increase competitive advantage; to create an innovative culture and environment; to entrench collaboration as a work practice; and to improve work efficiency [17]. Linking the individual perspective of knowledge to the organizational level, organizational knowledge creation theory is concerned with the processes which make available individual knowledge to the organizational knowledge system. This knowledge processes consist of several steps, starting with the creation of knowledge followed by the use of knowledge, the transfer and sharing of knowledge, and the storage and retrieval for further use. A crucial and difficult step in the organizational knowledge process is the conversion of tacit knowledge into explicit knowledge. Tacit (implicit) knowledge is unarticulated and rooted in experience and intuition and tied to the senses. Explicit knowledge is uttered, can be formulated in sentences, has a universal character and is accessible through consciousness. Only explicit knowledge can be integrated in the organizational knowledge base [18]. In other words, it can be classified as either tacit or explicit. Tacit knowledge primarily resides in peoples' minds and it is relatively difficult to be expressed, codified and documented. In contrast, explicit knowledge is that which has been articulated, codified and formalized in some electronic or physical form. In general terms, knowledge, when viewed as an object, can be perceived to be any piece of idea, insight, know what, know-how or meaningful information that can be used to achieve an objective [16]. Researchers and practitioners have suggested a multitude of approaches to managing knowledge, most of which can be categorized broadly into codification and personalization approaches. In the codification strategy, individual knowledge is amalgamated, put in a cohesive context, and made centrally available to members of the organization via databases and data warehouses. The codification strategy uses a document-to-person approach on the premise that knowledge can be effectively extracted and codified [19] and emphasizes the capability to help create, store, share, and use an organization's explicitly documented knowledge. This strategy emphasizes codifying and storing knowledge. Typically, knowledge can be codified via information technology. Codified knowledge is more likely to be reused. The emphasis is on completely specified sets of rules about what to do under every possible set of circumstances. This strategy is referred also as system Strategy [8]. Knowledge management using this approach is highly structured as compared to the personalization approach that is semi-structured. The personalization approach does not impose a distinction between the knowledge and the knowledge provider. It recognizes the tacit dimension of knowledge and assumes that knowledge is shared mainly through direct person-to-person contacts. The role of information technology here is to facilitate communication between members of the organization through tools such as e-mail, group support systems, etc [19]. It emphasizes knowledge sharing via interpersonal interaction and utilizes dialogue through social networks including occupational groups and teams. It helps share knowledge through person-to-person contacts. This strategy attempts to acquire internal and opportunistic knowledge and share it informally. Knowledge can be obtained from experienced and skilled people and can be referred also as human strategy [8]. Another new KM strategy has been suggested on the classification which is dynamic KM strategy. The dynamic KM strategy integrates the conceptual scope of system and human-oriented KM (HKM) strategies [20].

\section{B. Analytic Network Process (ANP)}

For solving the interactions among elements, the analytic network process (ANP) as a relatively new MCDM method was proposed [21]. The ANP is a mathematical theory that can deal with all kinds of dependence systematically [22].

Since the ANP/AHP has been proposed by Saaty, it has been widely used to deal with the dependence and the feedback decision making. The method of the ANP can be described as follows. The first phase of the ANP is to compare the criteria in whole system to form the supermatrix. This is done through pairwise comparisons by asking "How 
much importance does a criterion have compared to another criterion with respect to our interests or preferences?', The relative importance value can be determined using a scale of 1-9 to represent equal importance to extreme importance. The general form of the super matrix can be described as follows:

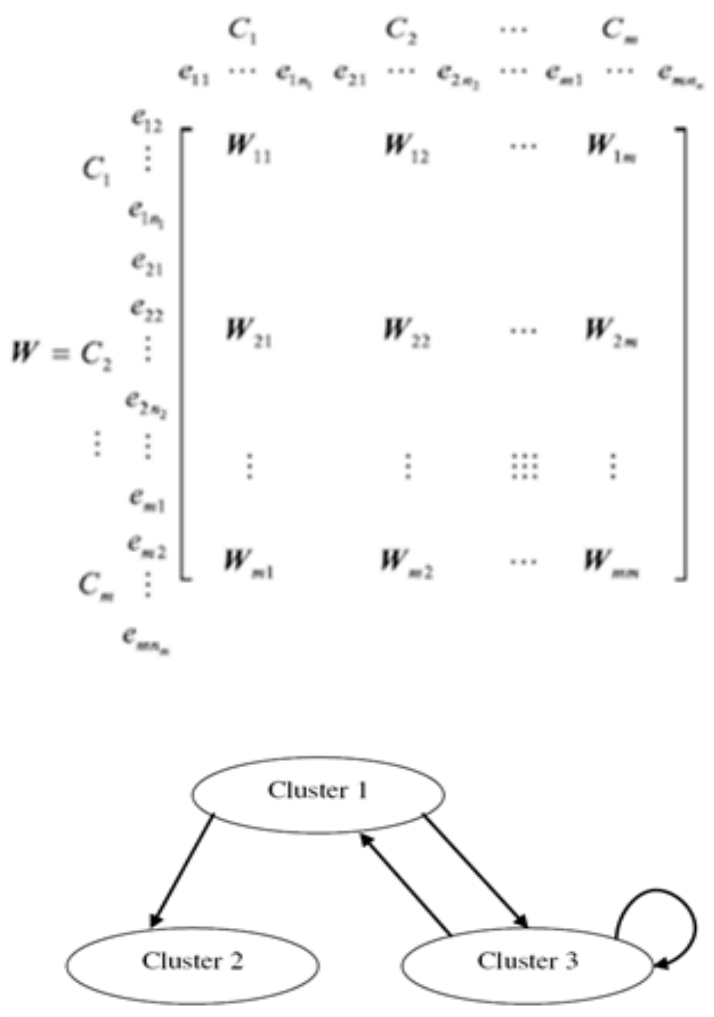

Fig 1. The Structure of the case 1.

where $\mathrm{Cm}$ denotes the $\mathrm{m} t h$ cluster, $\mathrm{e}_{\mathrm{mn}}$ denotes the $\mathrm{n} t h$ element in $\mathrm{m} t h$ cluster, and $\mathrm{W}_{\mathrm{ij}}$ is the principal eigenvector of the influence of the elements compared in the $\mathrm{j}$ th cluster to the $\mathrm{i}$ th cluster. In addition, if the jth cluster has no influence to the $\mathrm{i}$ th cluster, then $\mathrm{W}_{\mathrm{ij}}=0$.

Therefore, the form of the super matrix depends much on the variety of the structure. There are several structures which were proposed by Saaty including hierarchy, holarchy, suparchy, and intarchy to demonstrate how the structure affects the super matrix. Here, two simple cases, which both have three clusters, are used to display how to form the supermatrix based on the structures.

The supermatrix can be formed as the following matrix:

$$
\mathrm{W}=\quad \begin{gathered}
C_{1} \\
C_{1}
\end{gathered}\left[\begin{array}{ccc}
0 & C_{2} & C_{3} \\
C_{2} & 0 & w_{13} \\
w_{21} & 0 & 0 \\
w_{31} & 0 & w_{33}
\end{array}\right]
$$

In Fig 2. The second case is more complex than the first case.

Then,the supermatrix of the second case can be expressed as

$$
C_{1} \quad C_{2} \quad C_{3}
$$

$$
\mathrm{W}=\quad C_{2}\left[\begin{array}{ccc}
w_{11} & w_{12} & w_{13} \\
w_{21} & w_{22} & 0 \\
0 & w_{23} & 0
\end{array}\right]
$$

After forming the super matrix, the weighted super matrix is derived by transforming all columns sum to unity exactly. This step is much similar to the concept of Markov chain for ensuring the sum of these probabilities of all states equals to 1 . Next, we raise the weighted super matrix to limiting powers such as Eq. (1) to get the global priority vector or called weights.

$$
\lim _{\mathrm{k} \rightarrow \infty} W^{k}
$$

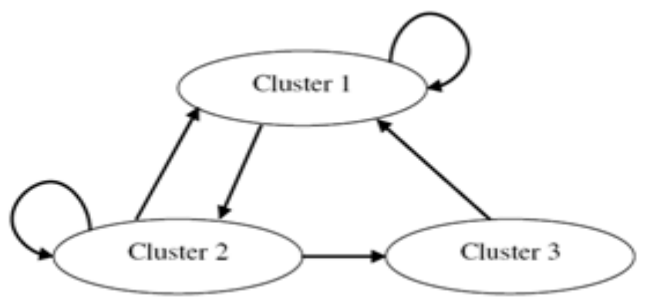

Fig 2. The structure of the case 2 .

In addition, if the super matrix has the effect of cyclicity, the limiting super matrix is not the only one. There are two or more limiting super matrices in this situation, and the Cesaro sum would be calculated to get the priority. The Cesaro sum is formulated as

$$
\lim _{k \rightarrow \infty}\left(\frac{1}{N}\right) \sum_{j=1}^{N} W_{j}^{k}
$$

to calculate the average effect of the limiting super matrix (i.e. the average priority weights) where $\mathrm{W}_{\mathrm{j}}$ denotes the $\mathrm{j}$ th limiting super matrix. Otherwise, the super matrix would be raised to large powers to get the priority weights. The detailed discussion of the mathematical processes of the ANP can refer to [23].

There are several MCDM methods which have been developed, such as the Elimination and Choice Translating Reality (ELECTRE), the Technique for Order Preference by Similarity to Ideal Solution (TOPSIS), and the Analytic Hierarchy Process (AHP). But these methods do not deal with the interdependences among elements. For dealing with the interdependences among elements, the ANP as a new MCDM method was proposed [21]. Specifically, the ANP is a new theory that extends the AHP to deal with dependence in feedback, and utilizes the supermatrix approach. Although both the AHP and the ANP derive ratio scale priorities by making paired comparisons of elements on a criterion, there are some differences between them[24]. The first difference is that the AHP is a special case of the ANP, because the ANP handles dependence within a cluster (inner dependence) and among different clusters (outer dependence). Secondly, the ANP is a nonlinear structure, while the AHP is hierarchical and linear with a goal at the top level and the alternatives in the bottom level [25]. Typically, the AHP model is a decision-making framework that assumes a unidirectional hierarchical relationship among decision levels. In the AHP model, the top element of the hierarchical structure is the overall goal for the decision model, and the hierarchy devolves to more specific attributes until a level of manageable decision criteria is met. By contrast, the ANP does not require this strictly hierarchical structure. The ANP model may consist of a single network or a number of 
networks[1]. In [25] has demonstrated several types of ANP models, such as: the Hamburger Model, the Car Purchase BCR model, and the National Missile Defense model. However, from the view point of [26], the ANP may simply be differentiated into two practical kinds of models: the Feedback System model and the Series System model (similar to the AHP model). In the feedback System model, clusters link one by one in turn as a network system. This kind of model can capture effectively the complex effects of interplay in human society, especially when risk and uncertainty are involved [24].

\section{Fuzzy Expert System}

A Fuzzy Expert System is simply an expert system that uses a collection of fuzzy membership functions and rules, instead of Boolean logic, to reason about data. Fuzzy Inference System (FIS) incorporates fuzzy inference and rule-based expert systems [16]. Fuzzy inference in this system refers to the use of computer programs to execute inference work resembling what humans do daily. The input constitutes some ambiguous linguistic semantics or unclear concepts for a specific event. Following the fuzzy inference mechanism, the output can be a fuzzy set or a precise set of certain features. Fuzzy inference infers the results from the existing knowledge base. 1) Fuzzy concept base: This contains the terminology and relevant predicate of a linguistic expression. Terminology is in the domain of the fuzzy set, possesses many pre-defined dismemberment values denoted by predicates. 2) Fuzzy proposition base: Membership functions accrue to the fuzzy proposition, which was induced from fuzzy concept base. There are numerous types of membership functions, such as S-shape, Z-shape, and P-shape, all easily definable with equations and parameters. For example, if the general fuzzy set is expressed as

$$
A=\left\{\left(x, \mu_{A 9}(X)\right)\right\}, x \in X\left(\mathrm{x}, \mu_{\mathrm{A}}(\mathrm{x})\right)
$$

where $\mathrm{i}$ denotes the membership function, and is a singleton, then a fuzzifier given by

$$
\mu(\mathrm{X})=1 /\left(1+\left(\mathrm{x} / \mathrm{K}_{2}\right)^{-\mathrm{K} 1}\right) \quad \mathrm{x} \in \mathrm{X}
$$

Produces an S-shaped curvature. K1 and K2 are called the exponential and denominational fuzzifiers, respectively. By having controllable parameters such as $\mathrm{K} 1$ and $\mathrm{K} 2$, adaptive fuzzy algorithms can be developed.3) Fuzzy rule base: The fuzzy proposition is then presented in IF-THEN format and constitutes the rule base. Specifically, a finite fuzzy logic implication statement in the rule base was described by a set of general fuzzy IF-THEN rules containing only the fuzzy logical AND operation, in the form 'IF a1 is A1 AND a2 is A2 THEN b1 is B1.'’4) Fuzzy strategy base: This contains the algorithms for computing the condition part and the conclusion part. A proposition might encompass many conditions. An appropriate fitness of a rule had to be found so that the conclusion can be drawn. This is carried out by a process of implication. A membership function that defines the implication relation can be expressed in a number of ways To illustrate the operation, we assume that we have the following simple conditional proposition (canonical rule):

IF $\mathrm{X}$ is $\mathrm{A}$ THEN $\mathrm{Y}$ is $\mathrm{B}$

The implication relation is defined by

$$
\mathrm{R}(\mathrm{x}, \mathrm{y})=\int_{r, y} \omega(\mathrm{x}, \mathrm{y}) /(\mathrm{x}, \mathrm{y})
$$

Where linguistic/fuzzy variable $\mathrm{X}$ and $\mathrm{Y}$ take the value of $\mathrm{A}$ and $\mathrm{B}$, respectively, and $\mathrm{l}(\mathrm{x}, \mathrm{y})$ is the membership function of the implication relation. The membership function is denoted by

$$
\mu(\mathrm{x}, \mathrm{y})=\mu_{\mathrm{A}}(\mathrm{x}) \Lambda \mu_{\mathrm{B}}(\mathrm{y}
$$

The symbol $\Lambda$ corresponds to intersection operation [17]. Numeric analysis approach of fuzzy system was first presented by Takagi and Sugeno and then a lot of studies have been made [27].

\section{RESEARCH Methodology}

To design the ANP model, the most important effective criteria on selection of knowledge management strategy have been specified. The results from running ANP model with the use of Super Decision software has showed that the most suitable knowledge management strategy is the Hybrid one.

In next step via an Fuzzy Expert system with two output variables, the best combination of the strategies has been determined. Two main steps for implementing ANP model and Fuzzy Expert system have been considered in our research ,as follows :

\section{A. To design ANP model includes:}

a) To identify the types of Knowledge management strategies according to the considered organization

b) To specify the effective variables on the selection of proper Knowledge management strategy

c) To present ANP model based on criteria and the selected alternatives

d) To do Pairwise comparison and to determine the best strategy

B. To design Fuzzy Expert system for identifying the suitable level of strategies in dynamic strategy consists of:

a) To Select the Input and output variables with the use of previous studies. Besides, meaningful linguistic states along with appropriate fuzzy sets for each variable should be selected.

b) To determine the membership functions for the variables.

c) To specify rules for clarifying the relations between Inputs and outputs.

d) To develop the Fuzzy Expert System via FIS Tool in MATLAB Software.

e) To identify the Knowledge Management Strategy based on the designed system.

f) The detail of models and the results has been explained in the rest of paper.

\section{RESUlt AND Discussion}

According to above mentioned steps, the effective variables on the selection of Knowledge Management strategy have been extracted from bank ${ }^{1}$ experts and the previous researches as Input variables [1,10,28]. These variables include: Top management Support, Communication, Culture and people, Incentives, Time and 
Cost. Selcuk [20] described them as follows:

Top management support (C1): Top management promotes the initial process of KM, supports ideas for improvement, and gives support and advice to the employees. Insufficient top management support and commitment can lead to potential sources of failure for the KM strategy.

Communication (C2): A knowledge sharing culture needs to be created for communication.

Culture and people (C3): KM strategy needs to be compatible with its organizational culture. A supportive culture encourages firm's employees to create and share knowledge within an organization.

Incentives (C4): reward system to motivate employees to share their knowledge.

Time (C5): It refers to the shortening the amount of time required to input and access information.

Cost (C6): It focuses on keeping the knowledge transaction costs as low as possible and/or under control.

Three kinds of Knowledge management strategy, Human-oriented Strategy (HOS) and System-oriented Strategy (SOS) and Dynamic Strategy (DS) are determined as ANP model outputs.

The structure of ANP model developed by Super Decision software, is shown in Fig.3.

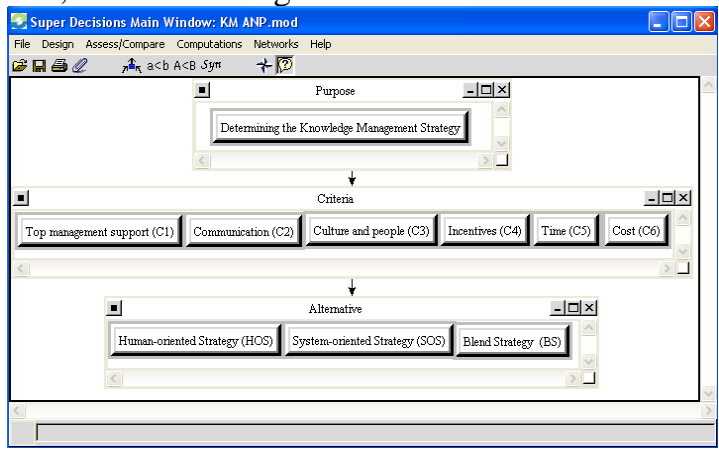

Fig.3. The structure of ANP model by Super Decision software

After doing pairwise comparisons according to expert's opinions ,the priority of criteria has been specified (Fig.4)

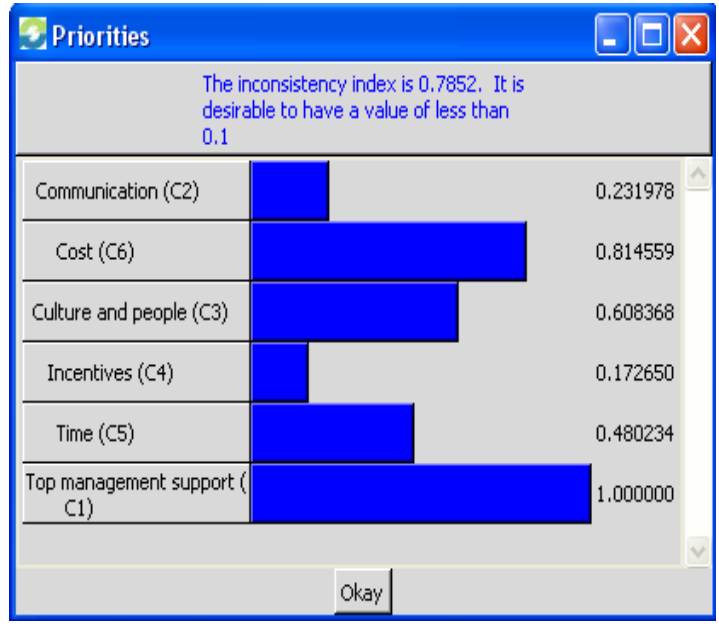

Fig.4. Priority of criteria after pairwise comparison

Final priority of knowledge management strategies are shown in Fig.5. According to obtained pairwise comparison Dynamic Strategy has higher priority than other strategies.

\footnotetext{
${ }^{1}$ Since the information of considered bank are confidential, The Authors have not been authorized to present The name of considered Bank.
}

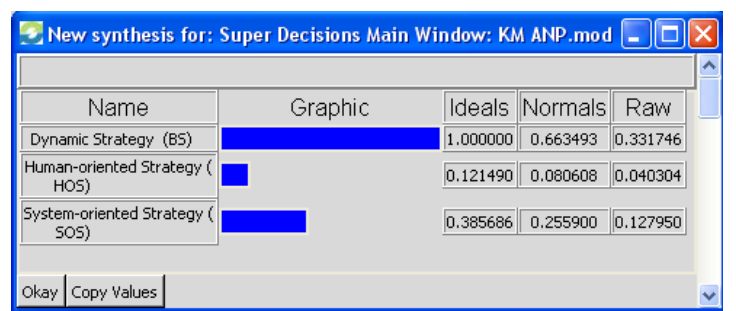

Fig.5. Priority of Knowledge Management Strategies after pairwise comparison

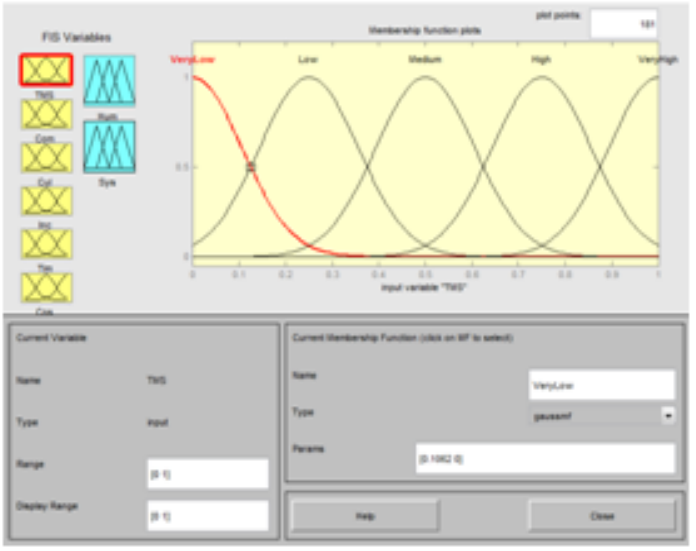

Fig 6. Five Gaussian Membership functions for Top management support

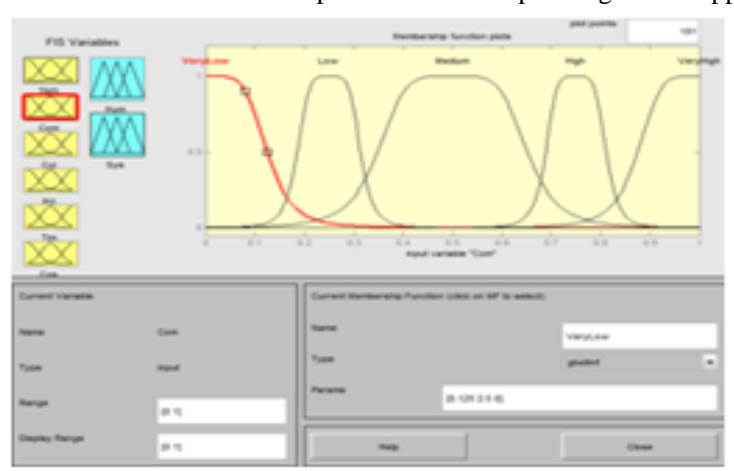

Fig 7. Five Gbell Membership functions for Communication

\begin{tabular}{|c|c|c|c|c|c|c|c|c|}
\multicolumn{10}{c}{ TABLE1. THE RULE OF FUZZY EXPERT SYSTEM } \\
\hline & C1 & C2 & C3 & C4 & $\begin{array}{c}\text { C } \\
5\end{array}$ & C6 & $\begin{array}{c}\text { HO } \\
\text { S }\end{array}$ & SOS \\
\hline 1 & M & M & H & M & L & M & H & L \\
2 & H & L & M & M & M & M & M & H \\
3 & M & H & H & M & M & H & H & M \\
4 & M & L & M & L & M & L & L & L \\
5 & H & M & L & H & M & H & H & L \\
6 & VH & H & VH & H & H & H & H & H \\
7 & H & VH & H & L & M & H & L & H \\
8 & L & M & M & M & M & VL & M & L \\
9 & H & M & H & L & H & M & L & M \\
10 & M & H & M & L & M & L & L & H \\
\hline
\end{tabular}

We need to know the percentage of works belonging to each strategy according to obtained results of ANP model and super decision software, for budgeting purpose and so on. In the other hand, determined Dynamic Strategy will specify the level of each strategy in the combination model.To response to this question, a fuzzy expert system has been developed. In this regard, Human-oriented Strategy (HOS) and System-oriented Strategy (SOS) have been considered as the outputs of Mamdani's Fuzzy Expert system. To design the system, the rules for determinning the relation between the variables have been identified.

Table 1 shows some of the obtained rules from the Bank 
Experts. The first rule shows when Top management supports KM in the medium level and Cultural situation is in the suitable level, Human-oriented Strategy is more preferable to System oriented one.

According to the experts' opinions, the Top management Support includes five Gaussian membership functions as: Very high (VH), High (H), Medium (M), Low (L), Very low (VL).The membership function of variables are shown in Fig 6-14.

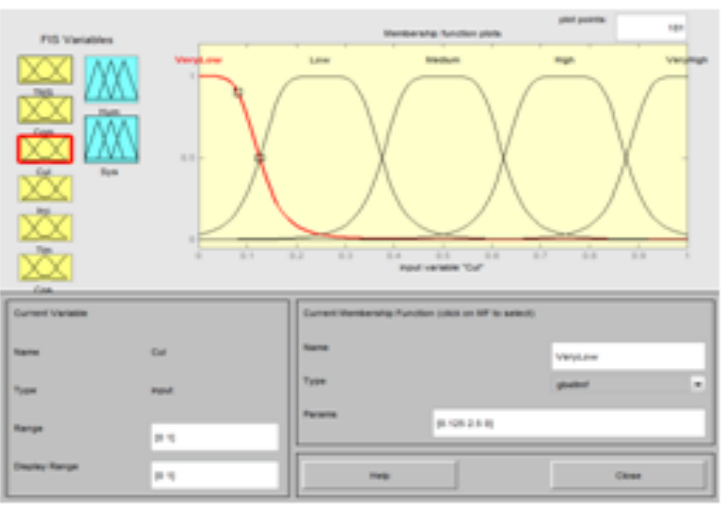

Fig 8. Five Gbell Membership function for Culture and people

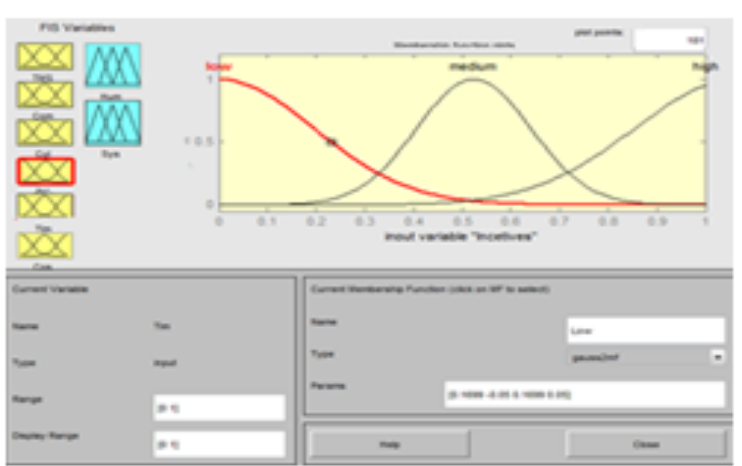

Fig 9. Three Gaussian Membership functions for Incentives

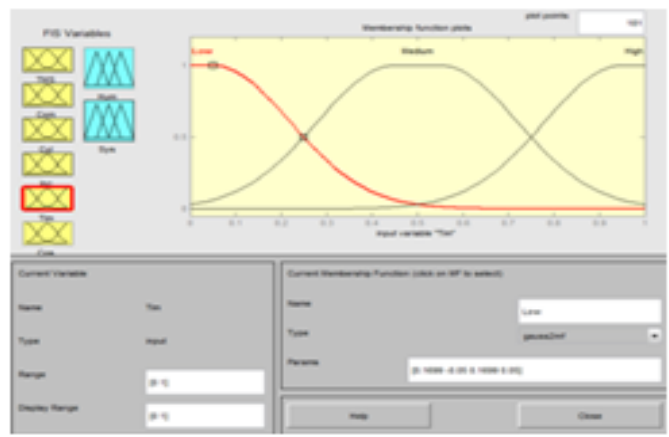

Fig 10. Three Gaussian2 Membership functions for Time

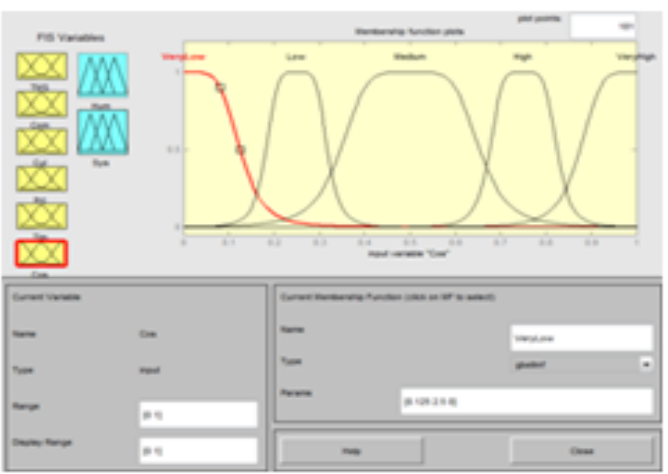

Fig 11. Five Gbell Membership functions for Cost

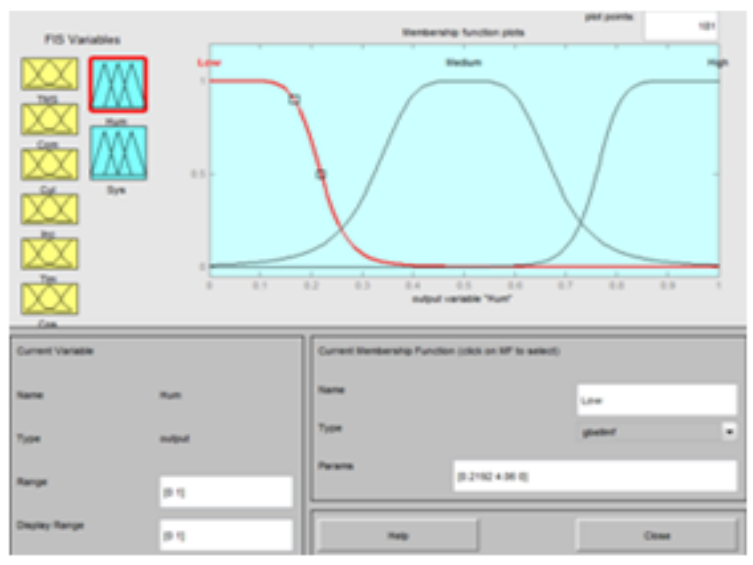

Fig 12. Three Gbell Membership functions for Human-oriented Strategy

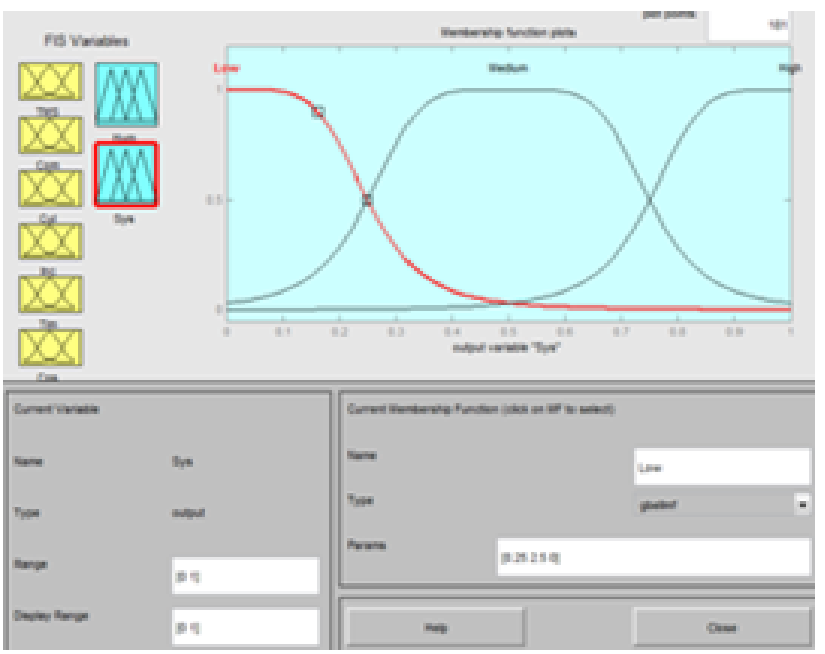

Fig 13. Three Gbell Membership functions for System-oriented Strategy

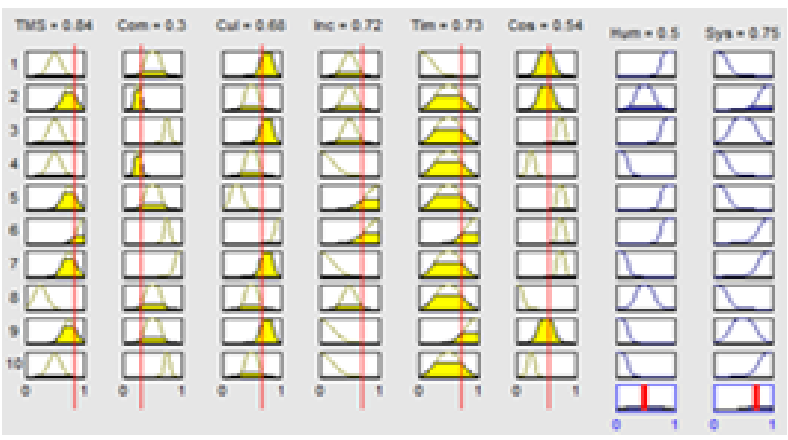

Fig14. Outputs of the designed System

Finally, with regard to acquired rules from the Experts and the Membership functions of variables, a Fuzzy Expert system has been designed via Fuzzy Inference System (FIS) in MATLAB (Appendix A).

The followings are the results of the designed Fuzzy Expert System for the Bank:

Top management support $=0.84 \quad$ Communication $=0.3$

Culture and people $=0.68$

Incentives $=0.72$

Time $=0.73$

Cost $=0.54$

Human oriented Strategy $=0.5 \quad$ System-oriented Strategy $=0.75$

This research is shown that Bank should pay more attention to the System-oriented strategy than Human-oriented strategy. 


\section{CONCLUSION}

In this research, the most effective criteria on selection of Knowledge Management strategy via ANP model, have been identified. The obtained results from ANP model via Super Decision software has showed that the most proper Knowledge Management strategy is the Dynamic model. In next step, by using a Fuzzy Expert system with two output variables, the most suitable combination level of each strategy has been specified. One of the advantages of this hybrid model, is selecting the best Knowledge Management strategy. Calculating the precision level of this hybrid model can be done as future researches.

\section{APPENDIX}

Here, some useful MATLAB commands to work with the proposed fuzzy inference system (FIS) which is based on Mamdani are presented:

[System]
Name='KM Strategy'
Type='mamdani'
Version=2.0
NumInputs=6
NumOutputs=2
NumRules=35
AndMethod='min'
OrMethod='max'
ImpMethod='min'
AggMethod='max'
DefuzzMethod='centroid'

\section{REFERENCES}

[1] W. W. Wu, Y.T. Lee,"Selecting knowledge management strategies by using the analytic network process", Expert Systems with Applications, 2007, 32, pp. 841-847.

[2] M.T. Hansen,N. Nohria,T. Tierney,"What's your strategy for managing knowledge?", Harvard Business Review,1999, 77(2), pp. 106-126.

[3] B. Choi, H. Lee,"An empirical investigation of $\mathrm{km}$ styles and their effect on corporate performance", Information \& Management,2003,40 (5), pp. 403-417.

[4] M. Schulz, L.A. Jobe,"Codification and tacitness as knowledge management strategies: an empirical exploration", Journal of High Technology Management Research, 2001, 12 (1), pp. 139-165.

[5] M.D. Santoro, P.E. Bierly,"Facilitators of knowledge transfer in university-industry collaborations: a knowledge-based perspective", IEEE Transactions on Engineering Management, 2006, 53 (4), pp. 495-507.

[6] J.M. Kamara, C.J. Anumba, P.M. Carrillo,"A CLEVER approach to selecting a knowledge management strategy",International Journal of Project Management,2002, 20(3), pp. 205-211.

[7] B. Choi, K. S.K. Poon, J.G. Davis,"Effects of knowledge management strategy on organizational performance:Acomplementarity theory-based approach ",2008, Omega 36,pp.235 - 251.

[8] B. Choi, H. Lee, "Knowledge management strategy and its link to knowledge creation process" , Expert Systems with Applications, 2002,23, pp.173-187
[9] T.H. Chang,T.H. Wang,"Using the fuzzy multi-criteria decision making approach for measuring the possibility of successful knowledge", Management. Information Sciences, 2009,179,pp. 355-370.

[10] W. W. Wu ,"Choosing knowledge management strategies by using a combined ANP and DEMATEL approach", Expert Systems with Applications,2008, 35,pp. 828-835.

[11] S. H. Chung, A. H. I. Lee, W. L. Pearn, " Analytic network process (ANP) approach for product mix planning in semiconductor fabricator". International Journal of Production Economics, 2005, 96(1), pp.15-36.

[12] K. Coulter, J,Sarkis. “ Development of a media selection model using the analytic network process". International Journal of Advertising.2005, 24(2), pp. 193-216.

[13] C. Kahraman, T. Ertay, G.Buyukozkan, “ A fuzzy optimization model for QFD planning process using analytic network approach". European Journal of Operational Research,2006, 171(2), pp.390-411.

[14] F. Y. Partovi, "An analytic model for locating facilities strategically". Omega, 2006,34(1), pp. 41-55.

[15] D.Tesfamariam, B.Lindberg," Aggregate analysis of manufacturing systems using system dynamics and ANP". Computers and Industrial Engineering, 2005, 49(1), pp.98-117.

[16] K. Yew Wonga, E. Aspinwall,"Development of a knowledge management initiative and system: A case study",Expert Systems with Applications, 2006, 30, pp.633-641.

[17] M. Plessis,"Drivers of knowledge management in the corporate environment", International Journal of Information Management, 2005,25(3), pp.193-202.

[18] F. Lindner, A. Wald, "Success factors of knowledge management in temporary organizations", International Journal of Project Management, 2010, In press.

[19] K. Desouza, R. Evaristo, "Global Knowledge Management Strategies", European Management Journal, 2003, 21( 1), pp. 62-67.

[20] S. Percin,"Use of analytic network process in selecting knowledge management strategies", Management Research Review, 2010, 33(5), pp. 452-471.

[21] T.L.Saaty, " The analytic network process-decision making with dependence and feedback". Pittsburgh, 1996,PA: RWS Publications.

[22] T.L.Saaty, "The analytic network process: Dependence and feedback in decision making (Part 1)", In The 17th International Conference on Multiple Criteria Decision Making, 2004.

[23] R. Yu," Gwo-Hshiung Tzeng, A soft computing method for multi-criteria decision making with dependence and feedback", Applied Mathematics and Computation,2006, 180 , pp.63-75

[24] R.W.Saaty, " The analytic hierarchy process (AHP) for decision making and the analytic network process (ANP) for decision making with dependence and feedback". Creative Decisions Foundation.2003.

[25] T. L. Saaty, "Fundamentals of the analytic network process". The International Symposium on the Analytic Hierarchy Process, 1999, Kobe, Japan.

[26] E.Kinosita "From AHP to ANP". Operations Research of Japan, 2003, 48(9), pp.677-683.

[27] Y. S. Juang, S. S.Lin, H. P..Kao ,"Design and implementation of a fuzzy inference system for supporting customer requirements",Expert Systems with Applications, 2007, 32 (3), pp. 868-878.

[28] M. Martensson, "A critical review of knowledge management as a management pool". Journal of Knowledge Management,2000,4(3),pp. 204-216.

Ahmad Nadali, photograph and biography not available at the time ofpublishing.

Hamid Eslami Nosratabadi, photograph and biography not available at the time ofpublishing.

Sanaz Pourdarab, photograph and biography not available at the time of publishing. 